\title{
Fenofibrate in Primary Biliary Cirrhosis: A Pilot Study
}

\author{
E.N. Liberopoulos ${ }^{1}$, M. Florentin ${ }^{1,2}$, M.S. Elisaf ${ }^{1, *}$, D.P. Mikhailidis ${ }^{2}$ and E. Tsianos ${ }^{1}$ \\ ${ }^{I}$ Department of Internal Medicine, Medical School, University of Ioannina, Ioannina, 45110, Greece \\ ${ }^{2}$ Department of Clinical Biochemistry (Vascular Disease Prevention clinics), Royal Free campus, University College \\ London Medical School, University College London (UCL), Pond Street, London NW3 2QG, UK
}

\begin{abstract}
Background: Most patients with primary biliary cirrhosis (PBC) are treated with ursodeoxycholic acid (UDCA); however, some do not respond fully. PBC is also associated with dyslipidemia, but a link with vascular risk has not been confirmed.

Methods and Results: In this study we compared UDCA monotherapy with fenofibrate plus UDCA in PBC patients with incomplete biochemical response to UDCA monotherapy for $\geq 8$ months. Ten patients $(57.2 \pm 13.3$ years old $)$ with PBC and persistent elevations of liver enzymes after treatment with UDCA $(600 \mathrm{mg} / \mathrm{day})$ were randomized to continue UDCA (4 patients) or to receive micronized fenofibrate ( $200 \mathrm{mg} /$ day) plus UDCA (6 patients) for 8 weeks. Significant reductions in total cholesterol, triglycerides and non-high density lipoprotein cholesterol were observed in the combination treatment group. The serum activities of alkaline phosphatase, gamma-glutamyl transpeptidase and alanine aminotranferase also decreased in this group compared with baseline $(-32.6 \% ; \mathrm{p}=0.012,-44 \% ; \mathrm{p}=0.031$ and $-16.9 \% ; \mathrm{p}=0.029$, respectively). In contrast, no significant alterations in liver enzymes or lipid profile were observed in patients who continued UDCA monotherapy. The changes in the lipid and enzyme variables differed significantly $(p<0.03)$ between the 2 groups. Fenofibrate was well tolerated.

Conclusions: The administration of fenofibrate plus UDCA seems to be safe and may improve lipid and liver indices in patients with PBC who do not respond fully to UDCA monotherapy. Whether the improved lipid profile translates into a decreased risk of vascular events remains to be established.
\end{abstract}

Keywords: Fibrates, ursodeoxycholic acid, primary biliary cirrhosis, liver enzymes, dyslipidemia.

\section{INTRODUCTION}

Primary biliary cirrhosis (PBC) is a chronic cholestatic liver disease characterized by inflammation and destruction of interlobular bile ducts, which may eventually lead to liver fibrosis, biliary cirrhosis and subsequently hepatic failure and death [1]. Although there is no established aetiology for the disease, PBC has been attributed to autoimmunity primarily due to its association with autoantibodies [especially antimitochondrial antibodies (AMA)] and elevated levels of immunoglobulin M (IgM) [1].

Ursodeoxycholic acid (UDCA), the most commonly used drug for PBC,[2] may ameliorate cholestasis [2]. However, some patients do not achieve complete biochemical response with UDCA treatment [3]. Fibrates (mainly bezafibrate), which are potent ligands of peroxisome proliferator-activated receptor $\alpha(\operatorname{PPAR} \alpha)$, have been tested in patients with PBC with promising results [4-11]. However, there are scarce data on the effects fenofibrate (a commonly used fibrate) in PBC. Therefore, we conducted this pilot study to compare the effects of UDCA monotherapy with the combination of UDCA and fenofibrate in patients with $\mathrm{PBC}$ who

*Address correspondence to this author at the Department of Internal Medicine, Medical School, University of Ioannina, Ioannina 45110, Greece; Tel: +302651007509; Fax: +302651007016; E-mail: egepi@cc.uoi.gr had incomplete biochemical response following UDCA monotherapy for $\geq 8$ months.

PBC is associated with dyslipidemia. However, it has not been established if this represents an increased risk of cardiovascular disease (CVD) [12]. Therefore, using a fibrate in patients with PBC may potentially improve both PBC-related liver damage and vascular risk.

\section{MATERIALS AND METHODOLOGY}

\section{Participants}

Ten patients ( 2 men and 8 women, $57.2 \pm 13.3$ years old) with histologically confirmed PBC (stages I and II) were included in the present study. These patients had persistent elevations of liver enzyme activities [specifically: alkaline phosphatase (ALP) >3-fold upper limit of normal] after treatment with UDCA $(600 \mathrm{mg} /$ day $)$ for at least 8 months. Patients with known CVD, diabetes mellitus, cancer, renal disease (serum creatinine levels $>1.6 \mathrm{mg} / \mathrm{dL}$ ) and hypothyroidism [thyroid stimulating hormone $(\mathrm{TSH})>5 \mathrm{IU} / \mathrm{mL}$ ] were excluded from the study. Patients taking lipid lowering drugs or having stopped them less than 6 weeks before study entry, as well as those currently taking other drugs that affect lipid metabolism (e.g. beta-blockers, thiazide diuretics, contraceptive pills or steroids) were also excluded. The patients were randomized to continue open-label UDCA (4 patients) 
or receive combined treatment with micronized fenofibrate (200 mg/day) and UDCA (600 mg/day) for 8 weeks (6 patients). Patients were instructed to follow their usual diet. Compliance with medication was assessed by pill count. All participants gave their written informed consent prior to enrolment and the Ethics Committee of the University Hospital of Ioannina approved the study protocol.

\section{Laboratory Determinations}

The activities of ALP, aspartate aminotransferase (AST) alanine aminotransferase (ALT) gamma glutamyl transpeptidase $(\gamma \mathrm{GT})$ and amylase as well as the levels of glucose, total and conjugated bilirubin, uric acid, creatinine and the lipid profile [i.e. total cholesterol, high density lipoprotein cholesterol (HDL-C), low density lipoprotein cholesterol (LDL-C), non-HDL-C and triglycerides] were assessed at baseline before randomization and after 8 weeks of treatment. The samples were collected after an overnight fast and assayed in the laboratory of the University Hospital of Ioannina using an Olympus AU 600 analyzer (Olympus Diagnostica GmbH, Hamburg, Germany). Glucose was measured by the hexokinase method. LDL-C levels were calculated using the Friedewald equation (LDL-C = total cholesterol - HDL$\mathrm{C}$ - triglycerides/5 in $\mathrm{mg} / \mathrm{dL}$ ) and non-HDL-C was defined as total cholesterol - HDL-C.

\section{Statistical Analysis}

All parameters were checked for normality using the Kolmogorov-Smirnov test. All values are expressed as mean \pm standard deviation (SD) except for non-Gaussian distributed variables which are expressed as median (range). The paired-samples $t$-test (or Wilcoxon's rank test for nonGaussian parameters) was used for assessing the effect of treatment in each group. Analysis of covariance (ANOVA), adjusted for baseline values, was used for comparisons between treatment groups. Significance was defined as $\mathrm{p}<0.049$ due to multiple comparisons (x2) [13]. All analyses were carried out using the SPSS 15.0 statistical package for Windows (SPSS Inc., Chicago, Illinois).

\section{RESULTS}

Baseline characteristics of the participants did not differ between the 2 groups (Table 1). Body mass index (BMI) did not change during the study in either group.

The addition of fenofibrate to UDCA resulted in significant decreases in the levels of total cholesterol, triglycerides and non-HDL-C (Table 2). Furthermore, significant reductions in the serum activities of ALP $(-32.6 \%, \mathrm{p}=0.012), \gamma \mathrm{GT}$ $(-44 \%, \mathrm{p}=0.04)$ and ALT $(-16.9 \%, \mathrm{p}=0.029)$ compared with baseline were observed in the combination treatment group (Table 2). On the other hand, no significant alterations in liver enzyme activities and lipid profile were demonstrated in patients who continued on UDCA monotherapy (Table 2). The change in the lipid and liver enzyme variables differed significantly $(\mathrm{p}<0.03)$ between the 2 groups (Table 2$)$. No significant changes in glucose, total and conjugated bilirubin, uric acid, amylase, creatinine and AST compared with baseline were observed in the 2 treatment groups.

Fenofibrate was well tolerated by all patients.

\section{DISCUSSION}

Significant improvements in lipid profile and liver enzyme activities were demonstrated in PBC patients who had an incomplete biochemical response to UDCA monotherapy and now received combined fenofibrate+UDCA treatment. In contrast, no change in these parameters was noted in the patients who continued on UDCA monotherapy.

Several small studies have investigated the effects of fibrates, either alone or in combination with UDCA in patients with $\mathrm{PBC}[5-7,10,14,15]$. In a prospective pilot study 23 patients with $\mathrm{PBC}$ who had been treated with UDCA for 1-5 years were randomly assigned to continue UDCA treatment $(600 \mathrm{mg} /$ day, $\mathrm{n}=13)$ or receive UDCA $(600 \mathrm{mg})$ and bezafibrate $(400 \mathrm{mg})(\mathrm{n}=10)$ for 12 months [7]. Significant reductions in ALP, $\gamma \mathrm{GT}$ and $\operatorname{IgM}$ at $3(\mathrm{p}<0.05), 6,9$ and 12 months $(\mathrm{p}<0.01)$ were observed in the bezafibrate+UDCA treatment group. The decrease in ALP and IgM was more pronounced as compared with that in the UDCA monotherapy group [7]. Comparable results have been demonstrated in other studies with similar design $[4,11]$.

Two studies compared the effects of bezafibrate with that of UDCA in patients with PBC. In 1 study 24 patients were randomly assigned to receive bezafibrate $400 \mathrm{mg} /$ day $(\mathrm{n}=12)$ or UDCA $600 \mathrm{mg} /$ day $(\mathrm{n}=12)$ for 12 months [6]. Significant reductions in ALT, ALP, $\gamma \mathrm{GT}$ and IgM were demonstrated in both treatment groups $(\mathrm{p}<0.0001)$; these changes were more pronounced in patients who received bezafibrate [6]. In contrast, in the other study bezafibrate was equally effective with UDCA [15]. However, it is not known whether these effects were translated into histological improvement as no biopsies were performed in the aforementioned studies.

There is few data in literature regarding the long-term efficacy of bezafibrate in patients with $\operatorname{PBC}[5,10,16]$. Nakamuta et al. followed 3 patients with $\mathrm{PBC}$ who were treated with bezafibrate (400 mg/day) and UDCA (600 mg/day) for an average of 37.5 months after treatment with UDCA for more than 4 years [16]. The mean levels of ALT, $\gamma \mathrm{GT}$, IgM and the titer of AMA were decreased after treatment ( $\mathrm{p}=0.079$ for ALT and $\gamma \mathrm{GT}$ and $\mathrm{p}=0.043$ for $\operatorname{IgM}$ vs baseline) [16].

Similar results in biochemical parameters along with histological changes were observed in 3 women with PBC after bezafibrate administration for 3, 4 and 5 years, respectively [5]. Substantial reductions in the levels of ALT, ALP, $\gamma \mathrm{GT}, \mathrm{IgM}$ and AMA, as well as a significant increase in apolipoprotein A-II levels were demonstrated in all patients. Furthermore, 2 of the 3 patients exhibited histological improvement while in the other patient there were no signs of deterioration [5]. This report indicated an association between biochemical and histological changes after bezafibrate treatment in PBC patients. In contrast, long-term bezafibrate treatment (6.5 and 6 years) did not lead to histological improvement in 2 patients with asymptomatic $\mathrm{PBC}$ and elevated liver enzymes [10].

There are some promising results in terms of fibrosis progression [9]. Seventeen patients with $\mathrm{PBC}$ who received bezafibrate (400 mg/day) and UDCA (600 mg/day) were followed for a mean of 59 months. The administration of 
Table 1. Baseline Characteristics of Study Participants

\begin{tabular}{|c|c|c|c|c|}
\hline $\begin{array}{l}\text { Mean duration of UDCA } \\
\text { monotherapy (months) }\end{array}$ & $8.6 \pm 0.8$ & $8.8 \pm 0.7$ & NS & \\
\hline Age (years) & $55.3 \pm 4.5$ & $54.8 \pm 7.3$ & NS & \\
\hline Sex (male/female) & $1 / 5$ & $1 / 3$ & NS & \\
\hline Body mass index $\left(\mathrm{kg} / \mathrm{m}^{2}\right)$ & $27.2 \pm 2.8$ & $27.9 \pm 3.1$ & NS & \\
\hline ALP (IU/L) & $195 \pm 73$ & $232 \pm 74$ & NS & $30-125$ \\
\hline AST (IU/L) & $42 \pm 15$ & $38 \pm 10$ & NS & $10-35$ \\
\hline Total bilirubin (mg/dL) & $0.70 \pm 0.14$ & $0.73 \pm 0.18$ & NS & $0.1-1.0$ \\
\hline Conjugated bilirubin (mg/dL) & $0.16 \pm 0.04$ & $0.17 \pm 0.07$ & NS & $0.0-0.3$ \\
\hline Uric acid (mg/dL) & $4.3 \pm 1.3$ & $4.1 \pm 1.2$ & NS & 3.4-8.0 (men) 2.4-6.1 (women) \\
\hline Creatinine $(\mathrm{mg} / \mathrm{dL})$ & $0.74 \pm 0.15$ & $0.76 \pm 0.12$ & NS & $0.6-1.2$ \\
\hline Amylase (IU/L) & $63 \pm 15$ & $68 \pm 16$ & NS & $30-90$ \\
\hline Total cholesterol (mg/dL) & $283 \pm 40$ & $302 \pm 45$ & NS & $110-200$ \\
\hline Triglycerides (mg/dL) & $145 \pm 67$ & $136 \pm 68$ & NS & $40-175$ \\
\hline
\end{tabular}

UDCA: ursodeoxycholic acid, ALP: alkaline phosphatase, AST: aspartate aminotransferase, ALT: alanine aminotransferase, $\gamma$ GT: gamma glutamyl transpeptidase, HDL-C: high density lipoprotein cholesterol, LDL-C: low density lipoprotein cholesterol, NS: not significant

Values are expressed as mean $\pm \mathrm{SD}$, except for $\gamma \mathrm{GT}$ which is expressed as median (range)

To convert bilirubin values to $\mu \mathrm{mol} / \mathrm{L}$ multiply by 17.1 . To convert uric acid values to $\mu \mathrm{mol} / \mathrm{L}$ multiply by 59.48 . To convert creatinine values to $\mu \mathrm{mol} / \mathrm{L}$ multiply by 88.4 . To convert cholesterol values to $\mathrm{mmol} / \mathrm{L}$ multiply by 0.026 . To convert triglyceride values to $\mathrm{mmol} / \mathrm{L}$ multiply by 0.0113 . To convert glucose values to mmol/L multiply by 0.05551 .

bezafibrate and UDCA was associated with significant reductions in hepatic fibrosis markers [9].

The activity of fenofibrate on $\operatorname{PPAR} \alpha$ is stronger than that of bezafibrate,[17] while fenofibrate exhibits more favourable effects on lipid profile compared with bezafibrate $[18,19]$. Furthermore, fenofibrate is a commonly used fibrate. However, there are only 3 studies on the effects of fenofibrate in $\mathrm{PBC}$ patients. In 1 study 7 patients with abnormal biochemical profile (elevated ALP, $\gamma \mathrm{GT}$ and $\mathrm{IgM}$ ) despite treatment with UDCA (600-900 $\mathrm{mg} /$ day) for 1-8 years were administered fenofibrate (150-200 $\mathrm{mg}$ /day) in addition to UDCA [20]. Treatment for 6 months resulted in reductions in the levels of these parameters in all 7 patients $(p<0.05$ vs baseline) [20]. In another study fenofibrate was added to UDCA for 12 weeks in 9 patients with asymptomatic PBC who failed to respond to UDCA monotherapy
[21]. The serum levels of ALP and IgM were significantly reduced after fenofibrate treatment $(\mathrm{p}<0.05)$, while the titer of AMA decreased in 4 of 9 patients. Lipid levels (total cholesterol, LDL-C, HDL-C and triglycerides) did not change significantly [21]. Consistently, in a recent study the addition of fenofibrate (134-200 mg/day) to UDCA for 23 months resulted in significant reductions in $\operatorname{IgM}(p=0.0015)$ and $\operatorname{ALP}(\mathrm{p}=0.0016)$ in 16 patients with lack of biochemical response (no significant decrease in ALP, ALT or IgM) to UDCA monotherapy (for a mean of 22.8 months) [22]. The findings of the aforementioned studies are consistent with the results of our study, suggesting that fenofibrate may be useful for the treatment of $\mathrm{PBC}$, especially in patients resistant to UDCA.

Data regarding the long-term effects of fenofibrate in $\mathrm{PBC}$ is not available. To the best of our knowledge only 
Table 2. Biochemical Biomarkers and Lipid Profile at Baseline (Before Randomization) and After 8 Weeks of Treatment

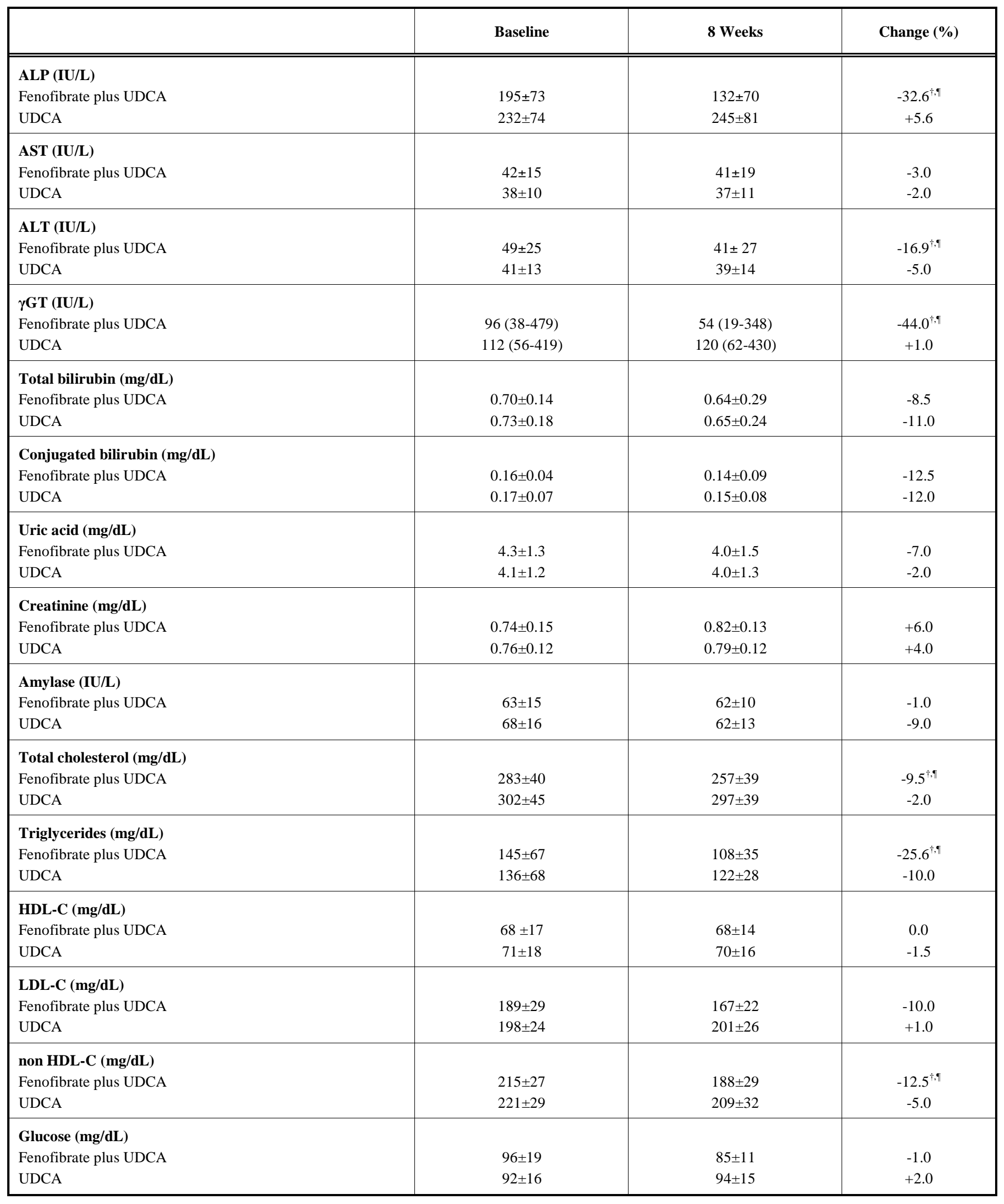

For abbreviations and conversions to SI units see Table 1

${ }^{\dagger} \mathrm{p}<0.049$ vs baseline

" $\mathrm{p}<0.03$ vs UDCA monotherapy

Values are expressed as mean $\pm \mathrm{SD}$, except for $\gamma \mathrm{GT}$ which is expressed as median (range). 
Nakamuta et al. reported the effects of treatment with fenofibrate (150 mg/day) and UDCA (600 mg/day) in 2 patients with $\mathrm{PBC}$ [16]. After an average of 37.5 months the mean levels of ALT, $\gamma \mathrm{GT}, \operatorname{IgM}$ and the titer of AMA were decreased $(\mathrm{p}=0.079$ for ALT and $\gamma \mathrm{GT}$ and $\mathrm{p}=0.043$ for IgM vs baseline) in both patients [16]. It is surprising that most of the studies discussed above did not report lipid levels.

The mechanisms by which fibrates may improve the histology in PBC have not been fully elucidated. These actions may be mediated via PPAR $\alpha$, the main target of fibrates, which are involved in the inflammatory and immunologic response. Fibrates inactivate leukotriene B4, a leukocyte activator, by activating $\beta$ and $\omega$ oxidation of fatty acids [23]. Furthermore, by suppressing the activation of the nuclear factor-kappaB (NF-kappaB), fibrates decrease the levels of interleukins 1 and 6 and cyclooxygenase 2 and subsequently the production of prostaglandin E2 and immunoglobulin [24]. The fibrate-associated increase in apolipoprotein A-II levels leads to suppressed expression of intercellular adhesion molecule 1 and vascular cell adhesion molecule 1 [25]. Of note, these adhesion molecules are involved in the inflammatory process in the biliary system [26]. It is, therefore, likely that these PPAR $\alpha$-mediated anti-inflammatory and immunomodulatory effects of fenofibrate contribute to the improvement of $\mathrm{PBC}$.

Biliary fibrosis in PBC results from cholestasis and intrahepatic accumulation of cytotoxic bile acids [1]. Fibrates facilitate the expression of the multiple drug resistance gene 3 which encodes the canalicular phospholipid translocator $[27,28]$. This receptor increases biliary phospholipid secretion and induces the formation of micelles with hydrophobic bile acids, thus inactivating the latter and protecting biliary epithelial cells $[27,28]$.

Furthermore, bile acids increase the expression of regulated on activation normal T-cell expressed and secreted (RANTES), which migrates memory type CD4+ Tlymphocytes to inflamed tissues, in the hepatocytes of patients with PBC [29]. Bezafibrate and fenofibrate decrease the chenodeoxycholic acid- and tumor necrosis factor- $\alpha$ induced mRNA expression and production of RANTES protein in human hepatoma cells $[30,31]$. These findings indicate that fibrates may inhibit inflammatory cell migration by RANTES to the liver in patients with PBC.

The 'hepatic' safety of fibrates has been confirmed by large clinical studies, while most of the adverse effects associated with fibrate treatment have been scarce [32].

It has not been clarified whether the lipid abnormalities in PBC are associated with increased risk for CVD [12]. Overall, data do not support a strong association between excess CVD risk and PBC. In this context, it has been suggested that hypercholesterolemia should be treated when other risk factors exist [33-35]. Statins, the mainstay of lipid lowering therapy, have been found to be safe and effective in improving the lipid profile in PBC patients, [36-40] overcoming the concerns about inducing elevations of liver enzymes [41]. However, statins do not seem to ameliorate cholestasis or progression of the disease;[38] none of these studies included histological findings.

Of note, fenofibrate has been associated with some beneficial effects on vascular events in patients with diabetes, [42] as well as with improvement (liver function tests and ultrasound) in non-alcoholic fatty liver disease [43]. However, as for statins, large, controlled, long-term studies are warranted to evaluate the efficacy of fibrates on histological improvement, symptom control and survival in patients with PBC.

Interestingly, the reduction in $\gamma \mathrm{GT}$ may be clinically relevant in terms of CVD risk. Indeed, evidence suggests that the serum $\gamma \mathrm{GT}$ concentration is an independent prognostic factor for CVD, including coronary heart disease, diabetes, stroke and CVD mortality [44-48]. Furthermore, $\gamma$ GT levels have been positively correlated with several other cardiovascular risk factors (e.g. LDL-C, triglycerides, uric acid, BMI, serum glucose, metabolic syndrome, smoking, hypertension and prehypertension),[46, 47, 49] some of which are commonly found in patients with $\mathrm{PBC}$.

The main limitations of this study are the small sample size and the lack of histological assessment after treatment. However, most of the studies that investigated the effects of fibrates in PBC were small and mainly only assessed biochemical markers. Therefore, there is no definite evidence to support the histological improvement or the delay of disease progression after treatment with fibrates. On the other hand, the design of this study is relevant to clinical practice, i.e. what options are available when patients fail to achieve a biochemical response while on UDCA monotherapy. In addition, most studies did not record the lipid levels. The issue of CVD risk in patients with $\mathrm{PBC}$ warrants further investigation.

Our data, along with others, suggest that the addition of fenofibrate is safe and probably a useful option in this setting.

\section{CONCLUSION}

UDCA is currently the most commonly used drug for the treatment of PBC. However, several patients do not achieve full biochemical response with UDCA and need supplementary treatment. Fibrates may improve the biochemical and probably the histological abnormalities in PBC. Fenofibrate, a widely available and potent fibrate, seems to be a safe alternative or adjunct therapeutic option and simultaneously improve the lipid profile (and possibly vascular risk) in patients with incomplete response to UDCA.

\section{ACKNOWLEDGEMENTS}

This study was conducted independently; no company or institution supported it financially. Some of the authors have given talks, attended conferences and participated in trials and advisory boards sponsored by various pharmaceutical companies. Matilda Florentin is supported by a grant from the Hellenic Atherosclerosis Society. 


\section{REFERENCES}

[1] Kaplan MM. Primary biliary cirrhosis. N Engl J Med 1996; 335 : 1570-80.

[2] Poupon R, Chretien Y, Poupon RE, Ballet F, Calmus Y, Darnis F. Is ursodeoxycholic acid an effective treatment for primary biliary cirrhosis? Lancet 1987; 1: 834-6.

[3] Goulis J, Leandro G, Burroughs AK. Randomised controlled trials of ursodeoxycholic-acid therapy for primary biliary cirrhosis: a meta-analysis. Lancet 1999; 354: 1053-60.

[4] Kanda T, Yokosuka O, Imazeki F, Saisho H. Bezafibrate treatment: a new medical approach for PBC patients? J Gastroenterol 2003; 38: 573-8.

[5] Kurihara T, Maeda A, Shigemoto M, Yamashita K, Hashimoto E. Investigation into the efficacy of bezafibrate against primary biliary cirrhosis, with histological references from cases receiving long term monotherapy. Am J Gastroenterol 2002; 97: 212-4.

[6] Kurihara T, Niimi A, Maeda A, Shigemoto M, Yamashita K. Bezafibrate in the treatment of primary biliary cirrhosis: comparison with ursodeoxycholic acid. Am J Gastroenterol 2000; 95: 29902.

[7] Nakai S, Masaki T, Kurokohchi K, Deguchi A, Nishioka M. Combination therapy of bezafibrate and ursodeoxycholic acid in primary biliary cirrhosis: a preliminary study. Am J Gastroenterol 2000; 95: 326-7.

[8] Ohmoto K, Mitsui Y, Yamamoto S. Effect of bezafibrate in primary biliary cirrhosis: a pilot study. Liver 2001; 21: 223-4.

[9] Ohmoto K, Yoshioka N, Yamamoto S. Long-term effect of bezafibrate on parameters of hepatic fibrosis in primary biliary cirrhosis. J Gastroenterol 2006; 41: 502-3.

[10] Yano K, Kato H, Morita S, Takahara O, Ishibashi H, Furukawa R. Is bezafibrate histologically effective for primary biliary cirrhosis? Am J Gastroenterol 2002; 97: 1075-7.

[11] Miyaguchi S, Ebinuma $\mathrm{H}$, Imaeda $\mathrm{H}$, et al. A novel treatment for refractory primary biliary cirrhosis? Hepatogastroenterology 2000; 47: 1518-21.

[12] Longo M, Crosignani A, Battezzati PM, et al. Hyperlipidaemic state and cardiovascular risk in primary biliary cirrhosis. Gut 2002; 51:265-9.

[13] Kusuoka H, Hoffman JI. Advice on statistical analysis for circulation research. Circ Res 2002; 91: 662-71.

[14] Kita R, Takamatsu S, Kimura T, Kokuryu H, Osaki Y, Tomono N. Bezafibrate may attenuate biliary damage associated with chronic liver diseases accompanied by high serum biliary enzyme levels. J Gastroenterol 2006; 41: 686-92.

[15] Iwasaki S, Ohira H, Nishiguchi S, et al. The efficacy of ursodeoxycholic acid and bezafibrate combination therapy for primary biliary cirrhosis: A prospective, multicenter study. Hepatol Res 2008; 38: 557-64.

[16] Nakamuta M, Enjoji M, Kotoh K, Shimohashi N, Tanabe Y. Longterm fibrate treatment for PBC. J Gastroenterol 2005; 40: 546-7.

[17] Willson TM, Brown PJ, Sternbach DD, Henke BR. The PPARs: from orphan receptors to drug discovery. J Med Chem 2000; 43: 527-50.

[18] Milionis HJ, Elisaf MS, Mikhailidis DP. Treatment of dyslipidaemias in patients with established vascular disease: a revival of the fibrates. Curr Med Res Opin 2000; 16: 21-32.

[19] Feussner G, Kurth B, Lohrmann J. Comparative effects of bezafibrate and micronised fenofibrate in patients with type III hyperlipoproteinemia. Eur J Med Res 1997; 2: 165-8.

[20] Ohira H, Sato Y, Ueno T, Sata M. Fenofibrate treatment in patients with primary biliary cirrhosis. Am J Gastroenterol 2002; 97: 21479.

[21] Dohmen K, Mizuta T, Nakamuta M, Shimohashi N, Ishibashi H, Yamamoto K. Fenofibrate for patients with asymptomatic primary biliary cirrhosis. World J Gastroenterol 2004; 10: 894-8.

[22] Walker LJ, Newton J, Jones DE, Bassendine MF. Comment on biochemical response to ursodeoxycholic acid and longterm prognosis in primary biliary cirrhosis. Hepatology 2009; 49: 337-8.

[23] Devchand PR, Keller H, Peters JM, Vazquez M, Gonzalez FJ, Wahli W. The PPARalpha-leukotriene B4 pathway to inflammation control. Nature 1996; 384: 39-43.

[24] Staels B, Koenig W, Habib A, et al. Activation of human aortic smooth-muscle cells is inhibited by PPARalpha but not by PPARgamma activators. Nature 1998; 393: 790-3.
[25] Calabresi L, Franceschini G, Sirtori CR, et al. Inhibition of VCAM-1 expression in endothelial cells by reconstituted high density lipoproteins. Biochem Biophys Res Commun 1997; 238: $61-5$.

[26] Gulubova M, Vlaykova T, Manolova I, Hadjipetkov P, Popharitov A. Implication of adhesion molecules in inflammation of the common bile duct in patients with secondary cholangitis due to biliary obstruction. Hepatogastroenterology 2008; 55: 836-41.

[27] Chianale J, Vollrath V, Wielandt AM, et al. Fibrates induce mdr2 gene expression and biliary phospholipid secretion in the mouse. Biochem J 1996; 314 (Pt 3): 781-6.

[28] Smit JJ, Schinkel AH, Oude Elferink RP, et al. Homozygous disruption of the murine mdr2 P-glycoprotein gene leads to a complete absence of phospholipid from bile and to liver disease. Cell 1993; 75: 451-62.

[29] Hirano F, Kobayashi A, Hirano Y, Nomura Y, Fukawa E, Makino I. Bile acids regulate RANTES gene expression through its cognate NF-kappaB binding sites. Biochem Biophys Res Commun 2001; 288: 1095-101.

[30] Hirano F, Kobayashi A, Makino I. Inhibition of TNF-alphainduced RANTES expression in human hepatocyte-derived cells by fibrates, the hypolipidemic drugs. Int Immunopharmacol 2003; 3: 225-32.

[31] Hirano Y, Hirano F, Fujii H, Makino I. Fibrates suppress chenodeoxycholic acid-induced RANTES expression through inhibition of NF-kappaB activation. Eur J Pharmacol 2002; 448: 19-26.

[32] Florentin M, Liberopoulos EN, Mikhailidis DP, Elisaf MS. Fibrateassociated adverse effects beyond muscle and liver toxicity. Curr Pharm Des 2008; 14: 574-87.

[33] Solaymani-Dodaran M, Aithal GP, Card T, West J. Risk of cardiovascular and cerebrovascular events in primary biliary cirrhosis: a population-based cohort study. Am J Gastroenterol 2008; 103: 2784-8.

[34] Balmer ML, Dufour JF. Treatment of hypercholesterolemia in patients with primary biliary cirrhosis might be more beneficial than indicated. Swiss Med Wkly 2008; 138: 415-9.

[35] Longo M, Crosignani A, Podda M. Hyperlipidemia in Chronic Cholestatic Liver Disease. Curr Treat Options Gastroenterol 2001; 4: 111-4.

[36] Del Puppo M, Galli Kienle M, Crosignani A, et al. Cholesterol metabolism in primary biliary cirrhosis during simvastatin and UDCA administration. J Lipid Res 2001; 42: 437-41.

[37] Ritzel U, Leonhardt U, Nather M, Schafer G, Armstrong VW, Ramadori G. Simvastatin in primary biliary cirrhosis: effects on serum lipids and distinct disease markers. J Hepatol 2002; 36: 4548.

[38] Stojakovic T, Claudel T, Putz-Bankuti C, et al. Low-dose atorvastatin improves dyslipidemia and vascular function in patients with primary biliary cirrhosis after one year of treatment. Atherosclerosis 2009 Sep 6 [Epub ahead of print].

[39] Rajab MA, Kaplan MM. Statins in Primary Biliary Cirrhosis: Are They Safe? Dig Dis Sci 2009 Oct 1 [Epub ahead of print].

[40] Lewis JH, Mortensen ME, Zweig S, Fusco MJ, Medoff JR, Belder R. Efficacy and safety of high-dose pravastatin in hypercholesterolemic patients with well-compensated chronic liver disease: Results of a prospective, randomized, double-blind, placebocontrolled, multicenter trial. Hepatology 2007; 46: 1453-63.

[41] Stojakovic T, Putz-Bankuti C, Fauler G, et al. Atorvastatin in patients with primary biliary cirrhosis and incomplete biochemical response to ursodeoxycholic acid. Hepatology 2007; 46: 776-84.

[42] Keech A, Simes RJ, Barter P, et al. Effects of long-term fenofibrate therapy on cardiovascular events in 9795 people with type 2 diabetes mellitus (the FIELD study): randomised controlled trial. Lancet 2005; 366: 1849-61.

[43] Athyros VG, Mikhailidis DP, Didangelos TP, et al. Effect of multifactorial treatment on non-alcoholic fatty liver disease in metabolic syndrome: a randomised study. Curr Med Res Opin 2006; 22: 87383 .

[44] Strasak AM, Kelleher CC, Klenk J, et al. Longitudinal change in serum gamma-glutamyltransferase and cardiovascular disease mortality: a prospective population-based study in 76,113 Austrian adults. Arterioscler Thromb Vasc Biol 2008; 28: 1857-65.

[45] Ruttmann E, Brant LJ, Concin H, Diem G, Rapp K, Ulmer H. Gamma-glutamyltransferase as a risk factor for cardiovascular disease mortality: an epidemiological investigation in a cohort of 163,944 Austrian adults. Circulation 2005; 112: 2130-7. 
[46] Lee DH, Silventoinen $\mathrm{K}$, Hu G, et al. Serum gammaglutamyltransferase predicts non-fatal myocardial infarction and fatal coronary heart disease among 28,838 middle-aged men and women. Eur Heart J 2006; 27: 2170-6.

[47] Wannamethee SG, Lennon L, Shaper AG. The value of gammaglutamyltransferase in cardiovascular risk prediction in men without diagnosed cardiovascular disease or diabetes. Atherosclerosis 2008; 201: 168-75.
[48] Korantzopoulos P, Tzimas P, Kalantzi K, et al. Association between serum gamma-glutamyltransferase and acute ischemic nonembolic stroke in elderly subjects. Arch Med Res 2009; 40: 582-9.

[49] Lippi G, Targher G, Montagnana M, Salvagno GL, Guidi GC Relationship between gamma-glutamyltransferase, lipids and lipoprotein(a) in the general population. Clin Chim Acta 2007; 384: $163-6$.

Received: March 16, 2010

Revised: March 31, 2010

Accepted: April 02, 2010

(C) Liberopoulos et al.; Licensee Bentham Open.

This is an open access article licensed under the terms of the Creative Commons Attribution Non-Commercial License (http://creativecommons.org/licenses/ by-nc/3.0/) which permits unrestricted, non-commercial use, distribution and reproduction in any medium, provided the work is properly cited. 